\title{
Anti-inflammatory effect of hispidin on LPS induced macrophage inflammation through MAPK and JAK1/STAT3 signaling pathways
}

\author{
Ying-Hao Han ${ }^{1 \dagger}$, Dong-Qin Chen ${ }^{1 \dagger}$, Mei-Hua Jin ${ }^{1 \dagger}$, Ying-Hua Jin ${ }^{2}$, Jing Li ${ }^{1}$, Gui-Nan Shen ${ }^{1}$, Wei-Long Li ${ }^{1}$, \\ Yi-Xi Gong ${ }^{1}$, Ying-Ying Mao ${ }^{1}$, Dan-Ping Xie', Dong-Seok Lee ${ }^{3}$, Li-Yun Yu' ${ }^{1}$, Sun-Uk Kim', Ji-Su Kim5, \\ Taeho Kwon ${ }^{5^{*}}$ (D, Yu-Dong Cui ${ }^{i^{*}}$ and Hu-Nan Sun ${ }^{1 *}$
}

\begin{abstract}
Severe inflammatory reactions caused by macrophage activation can trigger a systemic immune response. In the present study, we observed the anti-inflammatory properties of hispidin on LPS induced RAW264.7 macrophage cells. Our results showed that hispidin treatment significantly reduced the production of cellular NO, IL-6 and reactive oxygen species (ROS) while has not inhibitory effect on TNF-a productions. Excitingly, hispidin treatment retains the phagocytosis ability of macrophages which enabling them to perform the function of removing foreign invaders. Signaling studies showed, hispidin treatment dramatic suppressed the LPS induced mitogen activated protein kinases (MAPK) and JAK/STAT activations. In conclusion, our findings suggest that hispidin may be a new therapeutic target for clinical treatment of macrophages-mediated inflammatory responses.
\end{abstract}

Keywords: Hispidin, LPS, Macrophage, ROS, Signal transduction

\section{Introduction}

Inflammation is an important component of the innate immune response and can be caused by a variety of irritants, including invasive pathogenic microorganisms, harmful mechanical and chemical agents, cell fragmentation, and autoimmune responses [1]. On the other hand, the inflammatory response can increase microvascular caliber and vascular permeability, recruit white blood cells and release inflammatory mediators [2]. Although

\footnotetext{
*Correspondence: kwon@kribb.re.kr; cuiyudong@126.com;

sunhunan76@163.com

${ }^{\dagger}$ Ying-Hao Han, Dong-Qin Chen and Mei-Hua Jin contributed equally to this work

${ }^{1}$ College of Life Science \& Biotechnology, Heilongjiang Bayi Agricultural University, Daqing 163319, China

${ }^{5}$ Primate Resources Center, Korea Research Institute of Bioscience and Biotechnology (KRIBB), Jeongeup-si, Jeonbuk 56216, Republic of Korea

Full list of author information is available at the end of the article
}

the inflammatory response is initially occurs to protect the bodies from the damages, but the extensive studies have indicated that long-term and excessive inflammation is associated with various clinical manifestations such as arthritis [3], cardiovascular complications [4] and tumor progression [5]. Though the non-steroidal antiinflammatory drugs (NSAIDs) are widely used as antiinflammatory drugs [6], but long-term treatments can cause many adverse reactions in the body. Studies have shown that gastrointestinal bleeding and other complications, including peptic ulcers, are associated with longterm use of NSAIDs [7, 8].

Lipopolysaccharide (LPS), as a component of cell wall of Gram-negative bacteria, is one of the strongest inflammatory factors found in current research and is widely used in the induction of inflammation in animal and cell experiments [9-11]. Macrophages activated by LPS mainly involve three signaling pathways including 
NF-kB, MAPKs, JAK/STAT to produce amount of cytokines which are essential for procession of inflammatory responses [12-14]. By the way, Toll-like receptor 4 (TLR4) or CD14-specific receptors on cellular membrane can identify and bind to LPS, thereby activating these inflammatory signaling pathways in macrophages, which leads to release of tumor necrosis factor-alpha (TNF- $\alpha$ ), interleukin-6 (IL-6), nitric oxide (NO) and other proinflammatory mediators [15]. The accumulation of these inflammatory mediators results in increasing the tissue and cellular damages which can promote severe inflammatory responses in vivo and in vitro $[16,17]$ Therefore, efficiently inhibits the synthesis or release of these proinflammatory cytokines and mediators is an important strategy for the treatment of systemic immune response syndrome (SIRS).

The "Sanghuang" are well-known traditional medicinal fungi which are commonly used in East Asian countries [18]. It was reported that "Sanghuang" or its extracts have anti-oxidant, anti-bacterial, anti-tumor, regulate blood sugar, improve immune response and blood lipids functions in clinical treatments [19-21]. A paper published in Nature in 2019 showed that during the optimization of the "Sanghuang" strain, the obtained A67 strain had stronger antioxidant activity and inhibited tumor cell proliferation than the SH1 strain before optimization. Exploring the reasons for this, the author found that A67 contains more polyphenols than $\mathrm{SH} 1$, among which hispidin is the most abundant [22]. Hispidin, a polyphenolic substance, identified as 6-(3,4-dihydroxystyryl)-4-hydroxy-2-pyranone by Edwards in 1961, was first isolated from the fruiting body of I. hispidus by Zopf (1889) [23]. Subsequently, the researchers isolated hispidin and its derivatives from the ethanol extract of the fruiting bodies of Inonotus sp., I. xeronicus, P. linteus and Phellinus sp., fermentation products and the fruiting bodies of Fomitiporia ellipsoidea [24-26]. Hispidin isolated from the fruiting body of Gymnopilus spectabilis showed antioxidant activities, such as scavenging superoxide radicals. It's antioxidant activity was about 3-5 times that of water-soluble vitamin $E$, which is equivalent to that of BHA and caffeic acid [24]. It has also been reported that hispidin has anti-cancer, anti-bacterial and hypoglycemic regulatory functions [27-29]. Recently, Hong Jun Shao et al. has also demonstrated that hispidin can inhibited the macrophage mediated inflammatory response by down-regulating the NF- $\mathrm{kB}$ activations [30], however, the effect of hispidin on LPS induced macrophage ROS levels, phagocytosis as well as cytokines productions and it's molecular signaling mechanisms has remained unclear.

In the present study, we investigated the inhibitory effect of hispidin on the release of inflammatory mediators, cellular ROS level and phagocytic function in
RAW264.7 macrophages stimulated by LPS and explore the possible intracellular signaling regulated by hispidin on LPS induced macrophages.

\section{Materials and methods \\ Reagents and chemical}

Lipopolysaccharides (LPS) and hispidin were both purchased from Sigma (Sigma-Aldrich, St. Louis, MO, USA). The fetal bovine serum (FBS) and Dulbecco's modified Eagle's medium (DMEM) were purchased from Hyclone (General Electric Healthcare Life Sciences, Mississauga, Canada), penicillin streptomycin $(\mathrm{P} / \mathrm{S})$ was purchased from solarbio (Solarbio life sciences, Beijing, P. R. China).

\section{Cell culture}

The RAW264.7 macrophage cells (Shanghai BOGO Industrial Co., Ltd., Shanghai, China), was cultivated in DMEM supplemented with 10\% FBS and 1\% P/S (100 $\mathrm{U} / \mathrm{ml}$ and $100 \mathrm{mg} / \mathrm{ml}$, respectively). In the logarithmic growth phase, RAW264.7 macrophage cells maintained in DMEM at $37^{\circ} \mathrm{C}$ and $5 \% \mathrm{CO}_{2}$. After pretreatment with various concentrations of hispidin they were treated with $1 \mu \mathrm{g} / \mathrm{ml}$ LPS for the indicated time.

\section{Cell viability assay}

RAW264.7 macrophage cells were seeded into 96 well plates at a concentration of $4 \times 10^{3}$ cells per well, with different concentrations $(0 \mu \mathrm{g} / \mathrm{ml}$ to $20 \mu \mathrm{g} / \mathrm{ml})$ of hispidin treated $24 \mathrm{~h}$. Subsequently, a solution of $5 \mathrm{mg} / \mathrm{ml}$ 3-(4,5-dimethylthiazol-2-yl)-2,5-diphenyltetrazolium bromide (MTT; Sigma-Aldrich,) was added to each well and then incubated in an incubator $\left(37^{\circ} \mathrm{C}, 5 \% \mathrm{CO}_{2}\right)$ for $4 \mathrm{~h}$. After incubating $4 \mathrm{~h}$, remove the supernatant and add DMSO to dissolve formazan. Finally, use a UV MAX kinetic microplate reader (Molecular Devices, LLC) to measure the absorbance at $490 \mathrm{~nm}$.

\section{Griess method detect the production of NO}

The production of NO is reflected by the color rendering degree of nitrite generated after the reaction of culture medium with Griess reagent. Collect the culture supernatants and mix it with an equal volume of Griess reagent $[0.1 \%$ sulfanilamide, $0.1 \% \mathrm{~N}$-(1-naphthyl) ethylenediamine dihydrochloride and $2.5 \% \mathrm{H}_{3} \mathrm{PO}_{4}$ ]. Use a UV MAX kinetic microplate reader (Molecular Devices, LLC) to measure the absorbance at $540 \mathrm{~nm}$.

\section{Western blotting}

Harvested cells were lysed with protein lysis buffers (20 mM HEPES-OH, pH 7.0; $50 \mathrm{mM} \mathrm{NaCl} ; 10 \%$ glycerol and $0.5 \%$ Triton X 100) and extracted the total proteins.

Then, the total proteins were denatured for $5 \mathrm{~min}$ and using $12 \%$ sodium dodecyl sulfate-polyacrylamide gel 
electrophoresis to separate it and transferred into nitrocellulose membranes (EMD Millipore, Billerica, MA, USA). Using 5\% skimmed milk to block the membranes for $30 \mathrm{~min}$, at room temperature. They were then incubated with polyclonal rabbit anti iNOS, anti IкB- $\alpha$, anti $\beta$-actin; mouse monoclonal anti p-P38, anti p-JNK, anti p-ERK, anti P38, anti JNK, anti ERK primary antibodies (dilution, $1: 1000$ ) at $4{ }^{\circ} \mathrm{C}$ overnight. The membranes were washed five times with TBST (Tris buffered saline (TBS) containing Tween [150 mM NaCl, $10 \mathrm{mM}$ Tris $\mathrm{HCl}$ (pH 7.5) and $0.2 \%$ Tween-20]) and were subsequently incubated with horseradish peroxidase conjugated goat anti mouse IgG or anti rabbit IgG for $1 \mathrm{~h}$ at room temperature. After removing the excess antibodies by washing with TBST, specific conjugates were detected using a chemiluminescence detection system (GE Healthcare Life Sciences, Chalfont, UK) according to the manufacturer's protocol.

\section{Cytokine assays}

The concentration of IL- 6 and TNF- $\alpha$ in the cell culture supernatants were measured by using enzyme-linked immunosorbent assay (ELISA) kits for IL- 6 and TNF- $\alpha$. RAW264.7 macrophage cells $\left(2 \times 10^{5}\right.$ cells $)$ were plated into a 48-well plates and incubated with different concentrations of hispidin $(0 \mu \mathrm{g} / \mathrm{ml}$ to $20 \mu \mathrm{g} / \mathrm{ml})$ and $1 \mu \mathrm{g} /$ $\mathrm{ml}$ LPS for $24 \mathrm{~h}$. In order to determine the concentration IL- 6 of and TNF- $\alpha$ that had been released from the cells, the culture supernatants were collected and measured according to the manufacturer's protocols.

\section{Flow cytometry measure the phagocytosis and ROS}

Analysis the phagocytosis of macrophages by flow cytometry according to previously reported methods [31]. Alexa 488 conjugated BioParticles (Invitrogen; Thermo Fisher Scientific) were added to serum-free medium, sonicated, and the cells were incubated at $37{ }^{\circ} \mathrm{C}$ for $15 \mathrm{~min}$. Subsequently, the cells were washed three times with phosphate-buffered saline (PBS) and resuspended in $500 \mu \mathrm{l} \mathrm{PBS}$. The internalized fluorescence from 10,000 cells was immediately detected by using a FACScan flow cytometry (BD Biosciences, Franklin Lakes, NJ, USA). In order to detect ROS levels, cells were incubated with 10 mM DCFH-DA (Invitrogen; Thermo Fisher Scientific, Waltham, USA), a fluorescent indicator of ROS, which was incubated at $37{ }^{\circ} \mathrm{C}$ for $15 \mathrm{~min}$, and DCF-DA fluorescence intensity from 10,000 cells was measured using FACScan (BD Biosciences). The results were analyzed using WinMDI (Version 2.9, BD Biosciences) software.

\section{Statistical analysis}

The data are presented as the mean standard error of the mean. Differences between experimental groups were analyzed by one-way analysis of variance and a Tukey test. GraphPad Prism software version 4.0 (GraphPad Software, Inc., La Jolla, CA, USA) was used to analyze results. $P<0.05$ was considered to indicate a statistically significant difference.

\section{Results \\ Hispidin inhibits the production of NO in LPS-treated RAW264.7 macrophage cells}

The chemical structure of hispidin is presented in Fig. 1a. To ensure the effects of hispidin on macrophage cell viability, RAW264.7 macrophage cells were treated with hispidin at various concentrations $(0,1,510$ and $20 \mu \mathrm{g} /$ $\mathrm{ml}$ ) for $24 \mathrm{~h}$. As demonstrated in Fig. 1b, hispidin has no effect on macrophage cell viability. To clarify whether hispidin has anti-inflammatory properties, the NO levels in RAW264.7 macrophage cell culture supernatants were examined. The results showed that treatment with hispidin could dose- and time-dependently decrease the production of NO in LPS-treated RAW264.7 macrophage cells (Fig. 1c, d). Furthermore, the expression level of iNOS (an enzyme involved in NO production) protein was also observed with western blotting. As shown in Fig. 1e-h, hispidin treatment significantly down-regulated the LPS induced iNOS protein expression in a doseand time-dependent manner.

\section{Effect of hispidin on LPS induced production of IL-6 and TNF- $\alpha$ in RAW264.7 macrophage cells}

It was well known that TNF- $\alpha$ and IL- 6 are the major cytokines which produced by macrophage cells response to LPS stimulations. To verify whether the hispidin could influence the secretion of pro-inflammatory cytokines in LPS-treated RAW264.7 macrophage cells, the content of cytokines in cell culture supernatants was determined by ELISA. The results showed that LPS treatment dramatic increased the production of IL-6 which were significantly inhibited by treatment of hispidin in RAW264.7 macrophage cells (Fig. 2a, b). Unfortunately, hispidin treatment had no significant effect on LPS induced TNF- $\alpha$ production, though there has a slight inhibitory effect at $6 \mathrm{~h}$ (Fig. 2c, d).

\section{Effect of hispidin on LPS induced cellular ROS level and phagocytosis in RAW264.7 macrophage cells}

ROS has been proved to be an important signaling factor in LPS-treated macrophages. LPS-treated macrophages produce a large amount of ROS, and activate various signaling pathways to induce macrophage overactivation [32,33]. To examine whether hispidin affects the ROS levels in LPS-treated macrophages, RAW264.7 macrophage cells were pre-treated with hispidin $(20 \mu \mathrm{g} /$ $\mathrm{ml})$ for $30 \mathrm{~min}$, followed by treatment with LPS $(1 \mu \mathrm{g} /$ 
a<smiles>O=c1cc(O)cc(/C=C/c2ccc(O)c(O)c2)o1</smiles>

Hispidin

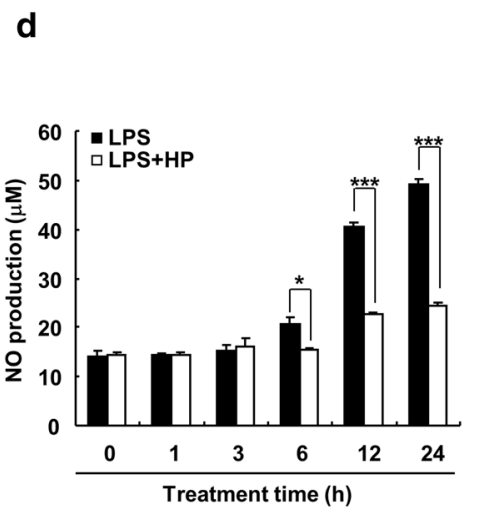

b

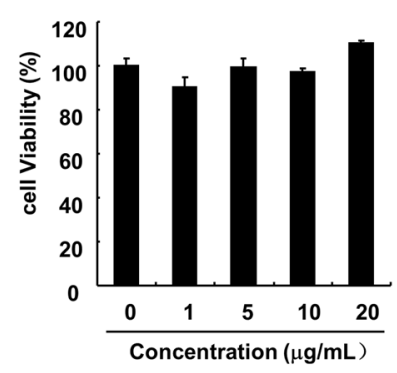

e

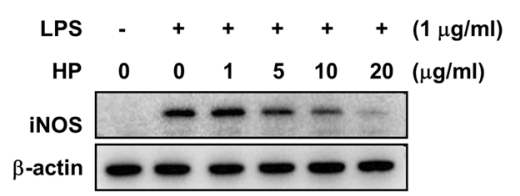

$\mathbf{f}$

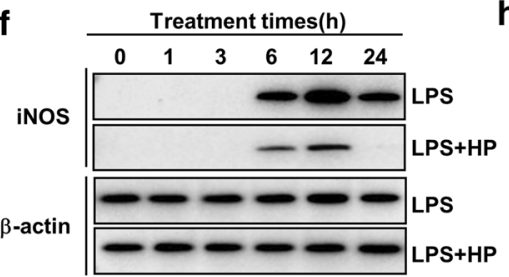

C

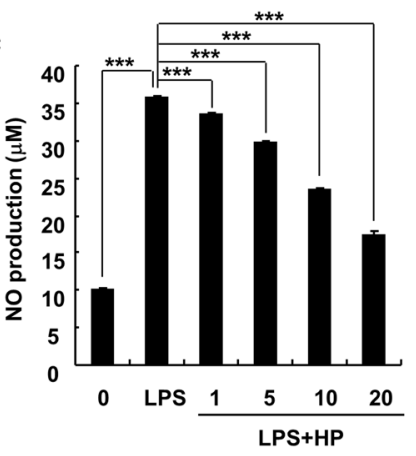

g

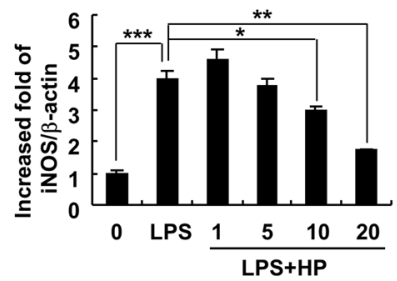

h

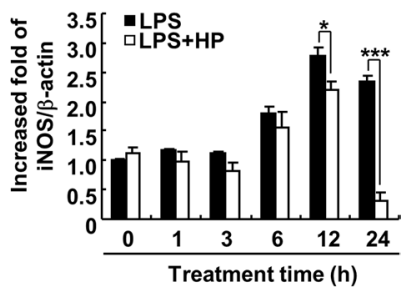

Fig. 1 Inhibitory effect of Hispidin on LPS induced NO prodution in RAW264.7 cells. a Chemical structure of Hispidin. b Cells were treated with different concentrations of Hispidin and incubated for $24 \mathrm{~h}$. Cell viability was measured by MTT assay. c, d RAW264.7 cells was pre-treated with indicated concentrations of Hispidin for $30 \mathrm{~min}$ followed by LPS $(1 \mathrm{\mu g} / \mathrm{ml})$ stimulation for $24 \mathrm{~h}$. The levels of NO production was measured by Griess reagent. e, f The protein expression levels of iNOS were detected by western blot by treatment with LPS and Hispidin combined with LPS in time- and dose-dependent manner. $\mathbf{g}, \mathbf{h}$ The related protein expression levels were represented with as the mean $\pm S D$. Data are presented as the mean standard error of the mean of three different samples. ${ }^{*} P<0.05,{ }^{* *} P<0.01$, and ${ }^{* *} P<0.001$

ml) for $24 \mathrm{~h}$. Cellular ROS levels were examined by flow cytometry and fluorescence microscopy. The results showed that LPS treatment upregulated the level of ROS in RAW264.7 macrophage cells, which were significantly down-regulated by pre-treatment with hispidin (Fig. 3ac). Phagocytosis, an important marker of macrophage activation, was also detected by hispidin treatment. The results showed that LPS treatment significantly increased the phagocytosis of RAW264.7 macrophage cells, but unfortunately, hispidin treatment could not affect the LPS induced macrophage phagocytosis (Fig. 3d-f).

\section{Hispidin exerts anti-inflammatory effects by suppresses LPS-induced MAPK and JAK1-STAT3 signaling pathways in RAW264.7 macrophage cells}

The NF-kB, MAPK and JAK1/STAT3 signaling pathways are crucial mediators of pro-inflammatory cytokines production in LPS-treated macrophages [14, 34-36]. To better define the underlying mechanism of anti-inflammatory effect of hispidin in LPS-treated RAW264.7 macrophage cell, the above three signaling pathways were detected. The results revealed that phosphorylation levels of ERK, JNK, and p38 were dramatic up-regulated by LPS treatments which were significantly inhibited by hispidin treatment in RAW264.7 macrophage cells (Fig. 4ad). Furthermore, the effect of hispidin on JAK/STAT signaling pathways were also examined in LPS induced RAW264.7 macrophage cells. The results revealed that hispidin treatment significantly down-regulated LPS induced JAK1 and STAT3 phosphorylation levels in RAW264.7 cells (Fig. 4e-g). These results comprehensively illustrates that hispidin inhibits LPS-mediated macrophage inflammatory response by extensively inhibiting intracellular signaling pathways.

\section{Discussion}

Inflammatory response plays a key role in the occurrence and development of various diseases, while macrophage is a target cell for regulating inflammatory related diseases. When stimulated by LPS, macrophage can activate 

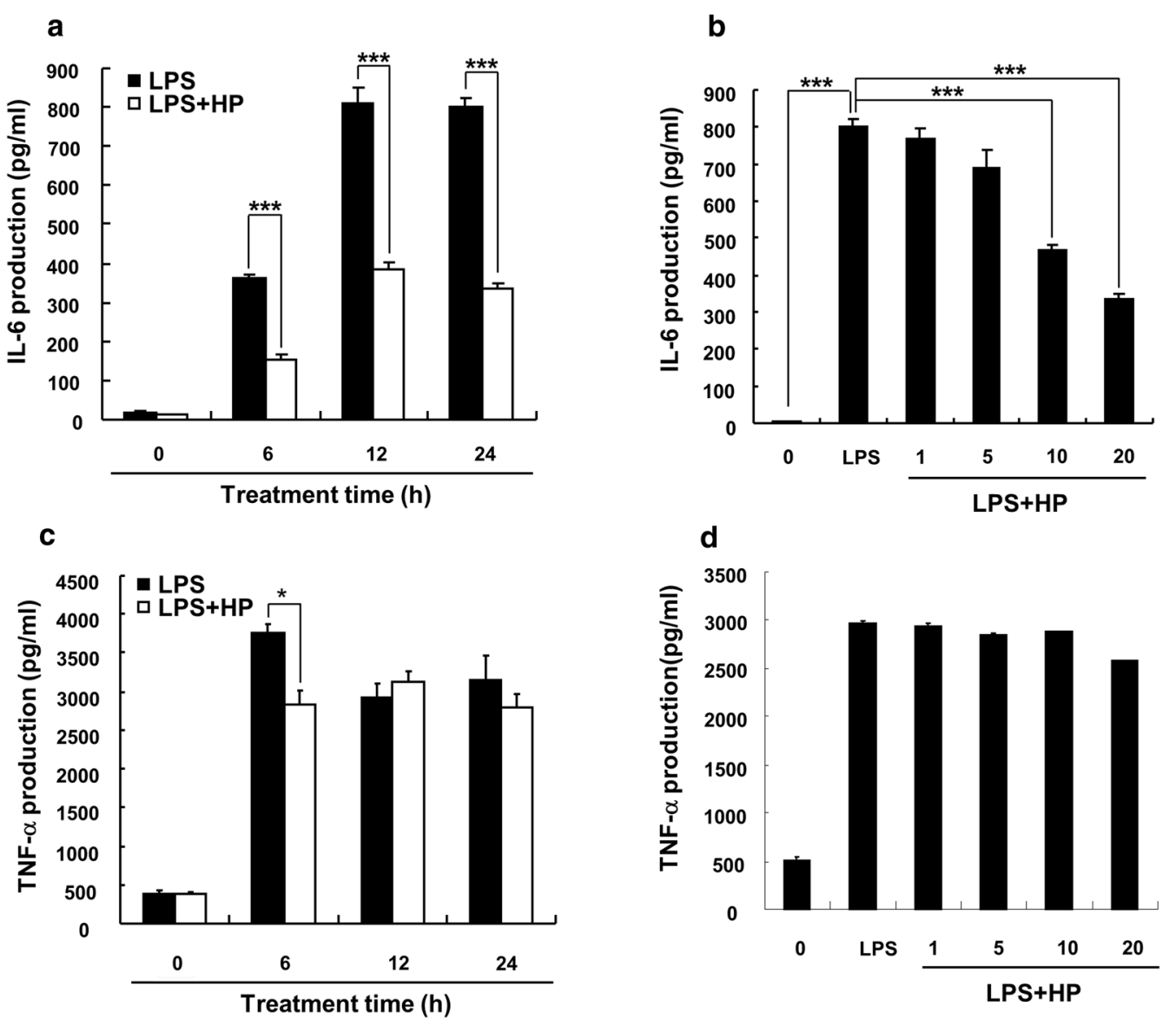

Fig. 2 Effect of Hispidin on LPS induced TNF- $a$ and IL-6 production in RAW264.7 macrophage cells. Cells were pre-treated with $20 \mu \mathrm{g} / \mathrm{ml} \mathrm{Hispidin}$ for $30 \mathrm{~min}$, followed by treatment with LPS $(1 \mu \mathrm{g} / \mathrm{ml})$ for the indicated durations. $\mathbf{a}, \mathbf{b} \mathrm{lL}-6$ and $\mathbf{c}$, $\mathbf{d}$ TNF-a productions were measured in the culture media by enzyme-linked immunosorbent assay (ELISA). Data are presented as the mean \pm standard error of the mean for three different samples. ${ }^{*} P<0.05$, and ${ }^{* * *} P<0.001$

related signal pathways NF- $\mathrm{B}$, MAPKs, JAK/STAT and then secrete a mass of NO, prostaglandin 2 , and proinflammatory cytokines, such as IL-6, TNF- $\alpha$, IL-1 $\beta$ [15].

Nitric oxide (NO) is a highly active diatomic molecule, which is catalyzed by NO synthase to produce $\mathrm{L}$-arginine in mammalian cells. As the second messenger of intracellular signal transduction, nitric oxide has a wide range of pathophysiological functions in the regulation of cardiovascular, digestive, immune and respiratory systems. Nitric oxide synthase includes structural constitutive nitric oxides synthase (cNOS) and inducible nitric oxide synthase (iNOS). iNOS is almost non-expressed in normal cells, but only in inflammation, hypoxia, tumor and other pathological states, followed by a large number of catalytic induced NO generations in a short period of time [37]. When macrophages are stimulated by substances such as LPS, the expression of iNOS is increased which in turn induces the secretion of large amounts of $\mathrm{NO}$ and promote the development of diseases such as inflammation [38]. In the process of inflammation, the pro-inflammatory cytokines, such as IL- 6 and TNF- $\alpha$ plays an important role in the pathogenesis of many diseases [17]. Therefore, effectively suppressing the secretion of NO and pro-inflammatory cytokines is one of the important strategies to control the inflammatory response. In the present study, treatment with hispidin could significantly decreased the production of NO and IL-6 as well as iNOS protein expression level in LPSinduced RAW264.7 macrophages cells, but not TNF- $\alpha$ (Figs. 1, 2). Our results strongly suggests that hispidin could play an essential roles in inhibiting the inflammatory response processed upon LPS stimulation. The different effects of compounds on inhibitory functions on TNF- $\alpha$ and IL- 6 productions have been reported in many researches. Our previous studies have also reported that $16 \alpha, 17 \alpha$-epoxypregnenolone-20-oxime could inhibit the LPS induced NO and IL- 6 productions but not TNF- $\alpha$ in BV2 microglia and RAW264.7 macrophage cells [39, 


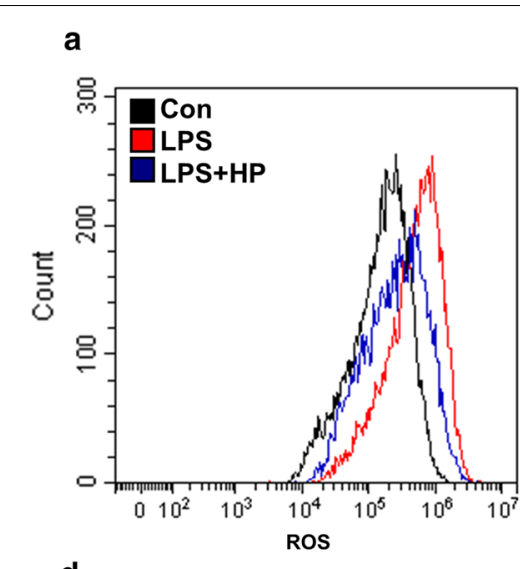

d

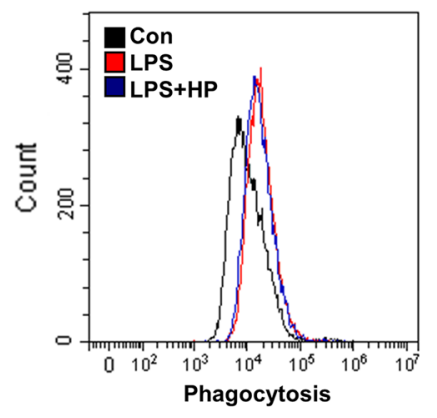

b
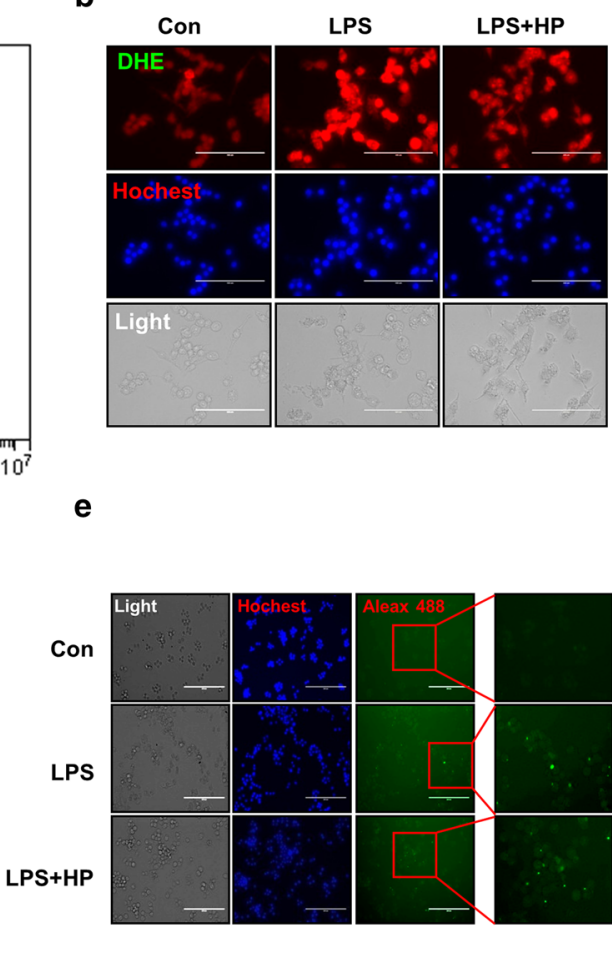

C

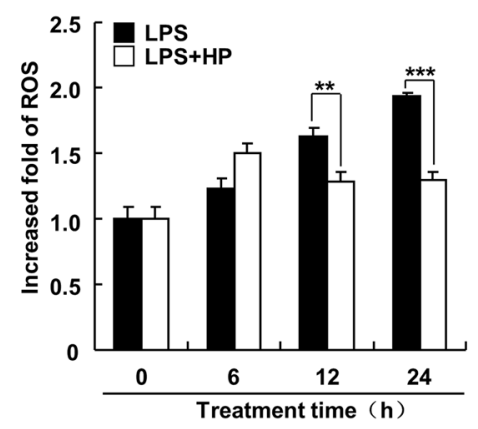

f
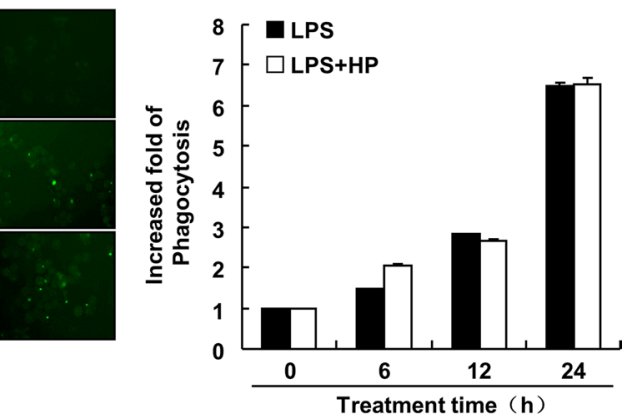

Fig. 3 Effect of Hispidin on LPS induced ROS and phagocytosis in RAW264.7 macrophage cells. Cells were pre-treated with $20 \mu \mathrm{g} / \mathrm{ml} \mathrm{Hispidin} \mathrm{for}$ $30 \mathrm{~min}$, followed by treatment with LPS $(1 \mathrm{\mu g} / \mathrm{ml})$ for the $24 \mathrm{~h}$. The level of cellular ROS and phagocytosis were detected using (a, d) flow cytometry (DCFH-DA for ROS and Alexa 488 for phagocytosis) and (b, e) fluorescence microscopy (DHE (red) for ROS and Hochest for nuclear; Alexa 488 for phagocytosis), scale bar $=200 \mathrm{~mm}$. c, f The ROS and phagocytosis were confirmed in a time-dependent durations with flow cytometry and the related data were presented as the mean \pm standard error of the mean for three different samples. ${ }^{* *} P<0.01,{ }^{* * *} P<0.001$

40]. Furthermore, is was also reported that knockdown of Peroxiredoxin I could efficiently affect the expression of IL-10 but not IL-1 $\beta$ and TNF- $\alpha$ in LPS induced RAW264.7 macrophage cells [41]. These findings suggests that there have different regulatory mechanisms on cytokines production upon LPS stimulation in macrophage cells, but possible mechanisms should be further studied.

Furthermore, the inhibitory effect of hispidin on cellular ROS level in LPS treated macrophages was also showed in our results (Fig. 3). It is well known that macrophages are activated with the increase of ROS and causes oxidative stress and amplify the inflammatory response [42]. It has been reported that exposure to ozone could up-regulate the expression of inflammatory mediator, cause lung inflammation; increase MDA level and GSH-PX activity; induce oxidative stress in the lung tissue; results in leading to worsening of asthma [43]. Our results about the inhibitory effect hispidin on ROS productions deeply demonstrates that the hispidin can be used as a potential anti-inflammatory drug candidate for treatment to LPS induced immune responses. When exogenous microbial pathogens invade the body, in order to eliminate them, macrophages will greatly improve their phagocytic ability after being activated. However, we examined the effect of hispidin on the phagocytic capacity of macrophages induced by LPS and found that hispidin had no effect on the phagocytic capacity of macrophages (Fig. 3d-f). The phenomenon that hispidin only inhibits the inflammatory response induced by LPS without affecting the phagocytosis capacity has aroused our interest, even though we have not studied the phenomenon in this study. After consulting the paper, we learned that when studying the reason that the Gram-positive bacteria Listeria monocytogenes and macrophages can coexist, it was found that when the bacteria infects J774 cells, the PKC family in the cells can translocate, resulting in less bacteria entering into the macrophages and escaping from the early phagocytes of macrophages. Hispidin, a PKC inhibitor [44], can inhibit the transfer of PKC $\beta$, allowing macrophages to engulf more bacteria and reduce the escape of bacteria from early phagosomes [45]. This suggests that whether hispidin does not affect the phagocytosis of LPS induced macrophages is also due 

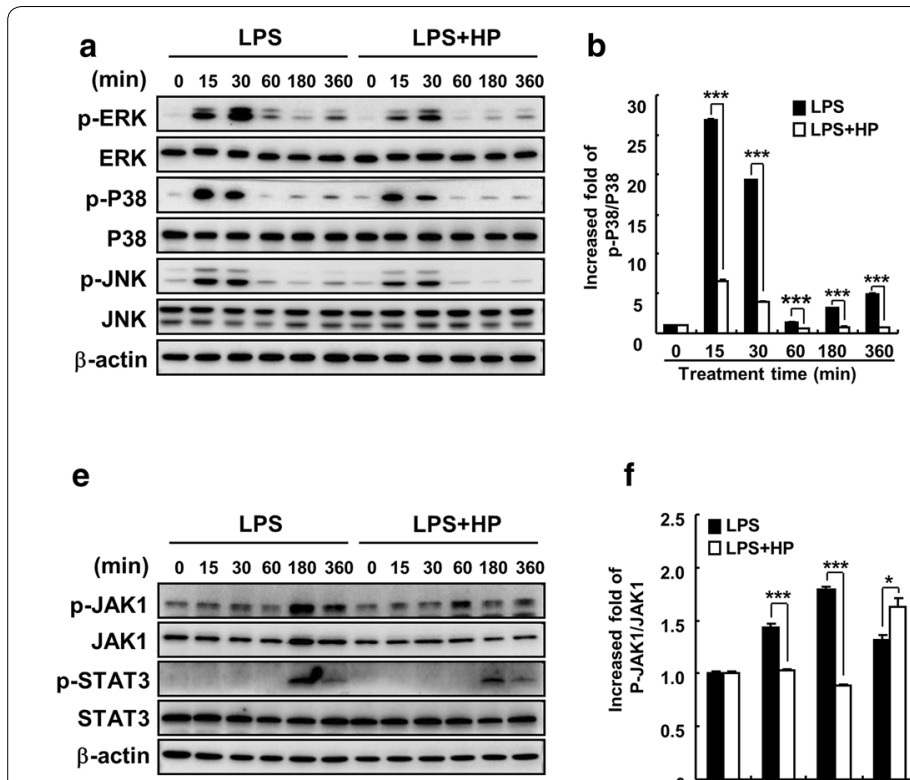

c

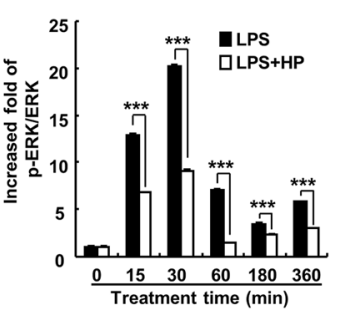

d

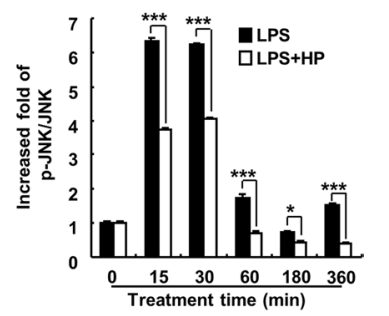

f

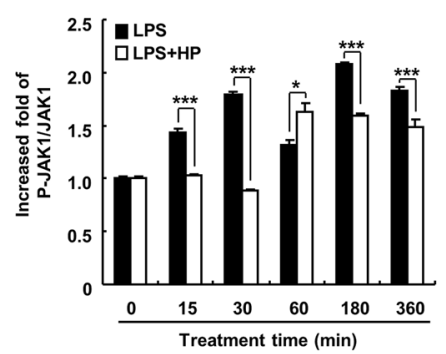

g

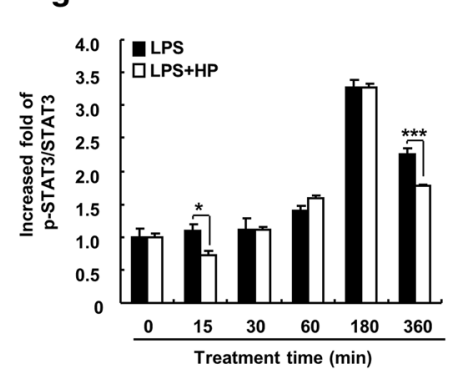

Fig. 4 Effect of Hispidin on LPS induced MAPK and JAK/STAT signaling pathways. Cells were pre-treated with $20 \mu \mathrm{g} / \mathrm{ml}$ Hispidin for $30 \mathrm{~min}$, followed by treatment with LPS $(1 \mathrm{\mu g} / \mathrm{ml})$ for the indicated durations. a Western blot analysis for p-ERK, ERK, p-P38, P38, p-JNK, JNK proteins expression. b-d The related protein expression levels were represented with as the mean $\pm S D,{ }^{*} P<0.05$ and ${ }^{* * *} P<0.001$. e Western blot analysis for $p-J A K 1, J A K 1$, p-STAT3, STAT3 proteins expression. $\mathbf{f}, \mathbf{g}$ The related protein expression levels were represented with as the mean $\pm S D,{ }^{*} P<0.05,{ }^{* * *} P<0.001$

to its ability to inhibit PKC in macrophages, which will be proved in subsequent experiments.

Sum up, our results also revealed that hispidin has the characteristics of high efficiency and low cytotoxicity in macrophage cells (Figs. 1, 2, 3, 4), therefore, we strongly believe that hispidin can be used as a candidate drug to inhibit inflammatory response and it can be widely used in future researches.

The major signaling pathways involved in macrophage activation are NF- $\mathrm{kB}$, MAPKs and JAK/STAT. In many studies of anti-inflammatory drugs, many of them can exert their anti-inflammatory effects by inhibiting the activation of the these signaling pathways $[14,34-36$, 46]. Recently, Hong Jun Shao et al. reported that hispidin could inhibit the activation of NF-kB in LPS-induced macrophages which results in suppressed the production of cytokines productions [30] suggests the anti-inflammatory properties of hispidin on LPS stimulated macrophage cells, but the molecular mechanisms remains unknown.

MAPKs are serine/threonine protein kinases, which mainly include c-Jun $\mathrm{N}$-terminal kinase (JNK), extracellular signal regulated kinase (ERK), and p38 MAPK [47]. ERK regulates cell proliferation and differentiation, JNK is involved in stress response, p38 is closely related to inflammation, apoptosis and other processes. MAPKs, which are relatively independent but interconnected between different signals, transmit signals from the outside of the cell to the inside through a three-stage kinase cascade reaction activated by MAPK kinase (MKKK), MAPK kinase (MKK), and MAPK [48]. MAPKs play an important regulatory roles in the expression and secretion of various inflammatory mediator, especially in the macrophages activated by LPS, which can produce ROS dependence phosphorylation [49-51]. In the present study, Hispidin can significantly inhibit the phosphorylation of P38, ERK, and JNK (Fig. 4a-d) suggest that the inhibitory effect of Hispidin on LPS induced macrophage activations were relayed on suppressing the MAPK signaling pathway which were known as ROS-dependent activated signaling cascades.

Cytokines signal transduction is mainly through mediating the JAK/STAT signaling pathway [52]. Cytokines bind to receptors on cell membrane, causing JAK to be phosphorylated which in turn phosphorylates downstream of STAT, eventually inducing a transcription response and increasing gene expression of related inflammatory mediators [53]. In the present study, we detected the phosphorylation of JAK1 and STAT3, and found that hispidin can slightly inhibit the phosphorylation of JAK1 and STAT3. Our results indicates that hispidin treatment can also reduce the production of inflammatory mediators by inhibiting the activation of JAK1/STAT3 (Fig. 4). 
Taken together, our studies suggest that hispidin exerts anti-inflammatory properties by attenuating the production of NO, IL-6 and intracellular ROS levels in LPS-induced macrophages by inhibiting the activation of MAPKs and JAK1/STAT3 signaling pathways. Excitingly, hispidin treatment retains the phagocytosis ability of macrophages, enabling them to perform the function of removing foreign invaders. Our findings may provide a new sight of hispidin as a therapeutic target for clinical treatment of macrophages-mediated inflammatory responses.

\section{Acknowledgements}

This work was supported by the scientific research team support plan of Heilongjiang Bayi Agricultural University (TDJH201904) and the University Nursing Program for Young Scholars with Creative Talents in Heilongjiang Province (CXRC2017016) and the Natural Science Foundation of Heilongjiang Province of China (QC2016012) and the "sanzong" project (ZRCPY201816), China.

\section{Authors' contributions}

Data curation: D-QC, Y-HJ, W-LL, Y-XG, YYM and D-PX. Formal analysis: Y-HH, M-HJ, G-NS, D-SL, S-UK and J-SK. Funding acquisition: JL, L-YY and TK. Supervision: TK, Y-DC and H-NS. All authors read and approved the final manuscript.

\section{Funding}

This research was supported by Basic Science Research Program through the National Research Foundation of Korea (NRF) funded by the Ministry of Education (2017R1D1A1B03028188). This study was supported by grants from the Korean Research Institute of Bioscience and Biotechnology Research Initiative Program (KRIBB) (KGM5162021, KGM4252021).

\section{Availability of data and materials}

The datasets used and analyzed in this study are available from the corresponding author upon reasonable request.

\section{Competing interests}

The authors declare that they have no competing interests.

\section{Author details}

${ }^{1}$ College of Life Science \& Biotechnology, Heilongjiang Bayi Agricultural University, Daqing 163319, China. ${ }^{2}$ Library and Information Center, Heilongjiang Bayi Agricultural University, Daqing 163319, China. ${ }^{3}$ KNU-Center for Nonlinear Dynamics, CMRI, School of Life Sciences, BK21 Plus KNU Creative BioResearch Group, College of Natural Sciences, Kyungpook National University, Daegu 41566, Republic of Korea. ${ }^{4}$ Futuristic Animal Resource \& Research Center, Korea Research Institute of Bioscience and Biotechnology (KRIBB), Cheongju-si, Chungcheongbuk-do 28116, Republic of Korea. ${ }^{5}$ Primate Resources Center, Korea Research Institute of Bioscience and Biotechnology (KRIBB), Jeongeup-si, Jeonbuk 56216, Republic of Korea.

Received: 20 January 2020 Accepted: 26 April 2020

Published online: 04 May 2020

\section{References}

1. Tracey KJ (2002) The inflammatory reflex. Nature 420(6917):853

2. Medzhitov R (2008) Origin and physiological roles of inflammation. Nature 454(7203):428

3. Xu N, Wang Y, Zhao S, Jiao T, Xue H, Shan F, Zhang N (2020) Naltrexone (NTX) relieves inflammation in the collagen-induced-arthritis (CIA) rat models through regulating TLR4/NFKB signaling pathway. Int Immunopharmacol 79:106056

4. Halaris A (2016) Inflammation-associated co-morbidity between depression and cardiovascular disease. In: Inflammation-associated depression: evidence, mechanisms and implications. ed. Springer, Berlin, pp 45-70
5. Mantovani A, Allavena P, Sica A, Balkwill F (2008) Cancer-related inflammation. Nature 454(7203):436

6. Antman EM, Bennett JS, Daugherty A, Furberg C, Roberts H, Taubert KA (2007) Use of nonsteroidal antiinflammatory drugs: an update for clinicians: a scientific statement from the American Heart Association. Circulation 115(12):1634-1642

7. Watanabe T, Fujiwara Y, Chan FK (2019) Current knowledge on nonsteroidal anti-inflammatory drug-induced small-bowel damage: a comprehensive review. J Gastroenterol 55(5):481-495

8. He Y, Chan EW, Man KK, Lau WC, Leung WK, Ho LM, Wong IC (2014) Dosage effects of histamine-2 receptor antagonist on the primary prophylaxis of non-steroidal anti-inflammatory drug (NSAID)associated peptic ulcers: a retrospective cohort study. Drug Saf 37(9):711-721

9. Sun HN, Feng L, Wang $A G$, Wang JY, Liu L, Jin MH, Shen GN, Jin $C H$, Lee DS, Kwon TH (2018) Peroxiredoxin I deficiency increases LPS-induced lethal shock in mice. Mol Med Rep 18(2):2427-2432

10. Henry CJ, Huang Y, Wynne AM, Godbout JP (2009) Peripheral lipopolysaccharide (LPS) challenge promotes microglial hyperactivity in aged mice that is associated with exaggerated induction of both proinflammatory IL-1 $\beta$ and anti-inflammatory IL-10 cytokines. Brain Behav Immun 23(3):309-317

11. Henry CJ, Huang Y, Wynne A, Hanke M, Himler J, Bailey MT, Sheridan JF, Godbout JP (2008) Minocycline attenuates lipopolysaccharide (LPS)-induced neuroinflammation, sickness behavior, and anhedonia. J Neuroinflamm 5(1):15

12. Lee S-B, Lee WS, Shin J-S, Jang DS, Lee KT (2017) Xanthotoxin suppresses LPS-induced expression of iNOS, COX-2, TNF- $a$, and IL-6 via AP-1, NF-KB, and JAK-STAT inactivation in RAW 264.7 macrophages. Int immunopharmacol 49:21-29

13. Kleinert $H$, Wallerath $T$, Fritz $G$, Ihrig-Biedert I, Rodriguez-Pascual $F$ Geller DA, Forstermann U (1998) Cytokine induction of NO synthase II in human DLD-1 cells: roles of the JAK-STAT, AP-1 and NF-KB-signaling pathways. Br J Pharmacol 125(1):193-201

14. Pan $X$, Cao X, Li N, Xu Y, Wu Q, Bai J, Yin Z, Luo L, Lan L (2014) Forsythin inhibits lipopolysaccharide-induced inflammation by suppressing JAK-STAT and p38 MAPK signalings and ROS production. Inflamm Res 63(7):597-608

15. Hailman E, Lichenstein HS, Wurfel MM, Miller DS, Johnson DA, Kelley M, Busse LA, Zukowski MM, Wright SD (1994) Lipopolysaccharide (LPS)binding protein accelerates the binding of LPS to CD14. J Exp Med 179(1):269-277

16. Cavaillon J (1994) Cytokines and macrophages. Biomed Pharmacother 48(10):445-453

17. Turner MD, Nedjai B, Hurst T, Pennington DJ (2014) Cytokines and chemokines: at the crossroads of cell signalling and inflammatory disease. Biochim Biophys Acta (BBA) Mol Cell Res. 1843(11):2563-2582

18. Han J-G, Hyun M-W, Kim CS, Jo JW, Cho J-H, Lee K-H, Kong W-S, Han S-K, Oh J, Sung G-H (2016) Species identity of Phellinus linteus (sanghuang) extensively used as a medicinal mushroom in Korea. J Microbiol 54(4):290-295

19. Friedman M (2016) Mushroom polysaccharides: chemistry and antiobesity, antidiabetes, anticancer, and antibiotic properties in cells, rodents, and humans. Foods 5(4):80

20. Xu X, Li J, Hu Y (2014) Polysaccharides from Inonotus obliquus sclerotia and cultured mycelia stimulate cytokine production of human peripheral blood mononuclear cells in vitro and their chemical characterization. Int Immunopharmacol 21(2):269-278

21. Fan L, Ding S, Ai L, Deng K (2012) Antitumor and immunomodulatory activity of water-soluble polysaccharide from Inonotus obliquus. Carbohydr Polym 90(2):870-874

22. Zhang H, Chen R, Zhang J, Bu Q, Wang W, Liu Y, Li Q, Guo Y, Zhang L, Yang $Y$ (2019) The integration of metabolome and proteome reveals bioactive polyphenols and hispidin in ARTP mutagenized Phellinus baumii. Sci Rep 9(1):1-12

23. Gill M, Steglich W. Progress in the chemistry of organic natural products. vol. 51: Berlin: Springer Science \& Business Media; 2012

24. Lee I-K, Cho S-M, Seok S-J, Yun B-S (2008) Chemical constituents of Gymnopilus spectabilis and their antioxidant activity. Mycobiology 36(1):55-59 
25. Zan L-F, Qin J-C, Zhang Y-M, Yao Y-H, Bao H-Y, Li X (2011) Antioxidant hispidin derivatives from medicinal mushroom Inonotus hispidus. Chem Pharm Bull 59(6):770-772

26. Nagatsu A, Itoh S, Tanaka R, Kato S, Haruna M, Kishimoto K, Hirayama H, Goda Y, Mizukami H, Ogihara Y (2004) Identification of novel substituted fused aromatic compounds, meshimakobnol A and B, from natural Phellinus linteus fruit body. Tetrahedron Lett 45(30):5931-5933

27. Chen W, He F-Y, Li Y-Q (2006) The apoptosis effect of hispolon from Phellinus linteus (Berkeley \& Curtis) Teng on human epidermoid KB cells. J Ethnopharmacol 105(1-2):280-285

28. Lee YS, Kang Y-H, Jung J-Y, Lee S, Ohuchi K, Shin KH, Kang I-J, Park JHY, Shin H-K, Lim SS (2008) Protein glycation inhibitors from the fruiting body of Phellinus linteus. Biol Pharm Bull 31(10):1968-1972

29. Halliwell B, Gutteridge J (1984) Oxygen toxicity, oxygen radicals, transition metals and disease. Biochem J 219(1):1

30. Shao HJ, Jeong JB, Kim KJ, Lee SH (2015) Anti-inflammatory activity of mushroom-derived hispidin through blocking of NF-kB activation. J Sci Food Agric 95(12):2482-2486

31. Liu Y, Hao W, Letiembre M, Walter S, Kulanga M, Neumann H, Fassbender K (2006) Suppression of microglial inflammatory activity by myelin phagocytosis: role of p47-PHOX-mediated generation of reactive oxygen species. J Neurosci 26(50):12904-12913

32. Ying J, Zhang M, Qiu X, Lu Y (2018) The potential of herb medicines in the treatment of esophageal cancer. Biomed Pharmacother 103:381-390

33. Parhizkar S, Zulkifli SB, Dollah MA (2014) Testicular morphology of male rats exposed to Phaleria macrocarpa (Mahkota dewa) aqueous extract. Iran J Basic Med Sci 17(5):384

34. Hu X, Herrero C, Li W-P, Antoniv TT, Falck-Pedersen E, Koch AE, Woods JM, Haines GK III, Ivashkiv LB (2002) Sensitization of IFN- $\gamma$ Jak-STAT signaling during macrophage activation. Nat Immunol 3(9):859

35. Su Y-W, Chiou W-F, Chao S-H, Lee M-H, Chen C-C, Tsai Y-C (2011) Ligustilide prevents LPS-induced iNOS expression in RAW 264.7 macrophages by preventing ROS production and down-regulating the MAPK, NF-KB and AP-1 signaling pathways. Int Immunopharmacol 11(9):1166-1172

36. Sharif O, Bolshakov VN, Raines S, Newham P, Perkins ND (2007) Transcriptional profiling of the LPS induced NF-KB response in macrophages. BMC Immunol 8(1):1

37. Martín MC, Martinez A, Mendoza JL, Taxonera C, Díaz-Rubio M, Fernández-Arquero M, Emilio G, Urcelay E (2007) Influence of the inducible nitric oxide synthase gene (NOS2A) on inflammatory bowel disease susceptibility. Immunogenetics 59(11):833-837

38. Janakiram NB, Rao CV (2012) iNOS-selective inhibitors for cancer prevention: promise and progress. Fut Med Chem 4(17):2193-2204

39. Sun HN, Han YH, Feng L, Jin CH, Han B, Liu L, Lee DS, Kwon TH, Li LG, Ge WZ (2016) 16a, 17a-epoxypregnenolone-20-oxime inhibits NO and IL-6 production in LPS-treated RAW264.7 cells. Mol Med Rep 13(6):4927-4933

40. Sun H-N, Jin M-H, Han B, Feng L, Han Y-H, Shen G-N, Yu Y-Z, Jin C-H, Lian Z-X, Lee D-S (2014) 16a, 17a-Epoxypregnenolone-20-oxime prevent LPSinduced NO production and iNOS expression in BV-2 microglial cells by inhibiting JNK phosphorylation. Biol Pharm Bull 37(7):1096-1102
41. Tae Lim Y, Sup Song D, Joon Won T, Lee YJ, Yoo JS, Eun Hyung K, Won Yoon J, Park SY, Woo Hwang K (2012) Peroxiredoxin-1, a possible target in modulating inflammatory cytokine production in macrophage like cell line RAW264.7. Microbiol Immunol 56(6):411-419

42. Ikwegbue PC, Masamba P, Oyinloye BE, Kappo AP (2018) Roles of heat shock proteins in apoptosis, oxidative stress, human inflammatory diseases, and cancer. Pharmaceuticals 11(1):2

43. Bao A, Yang H, Ji J, Chen Y, Bao W, Li F, Zhang M, Zhou X, Li Q, Ben S (2017) Involvements of p38 MAPK and oxidative stress in the ozone-induced enhancement of AHR and pulmonary inflammation in an allergic asthma model. Respir Res 18(1):216

44. Gonindard C, Bergonzi C, Denier C, Sergheraert C, Klaebe A, Chavant L, Hollande E (1997) Synthetic hispidin, a PKC inhibitor, is more cytotoxic toward cancer cells than normal cells in vitro. Cell Biol Toxicol 13(3):141-153

45. Wadsworth SJ, Goldfine H (2002) Mobilization of protein kinase C in macrophages induced by Listeria monocytogenes affects its internalization and escape from the phagosome. Infect Immun 70(8):4650-4660

46. Jang Y-J, Kim M-E, Ko S-Y (2011) Butanol extracts of Panax notoginseng suppress LPS-induced MMP-2 expression in periodontal ligament fibroblasts and inhibit osteoclastogenesis by suppressing MAPK in LPSactivated RAW264.7 cells. Archiv Oral Biol 56(11):1319-1327

47. Cano E, Mahadevan LC (1995) Parallel signal processing among mammalian MAPKs. Trends Biochem Sci 20(3):117-122

48. Roux PP, Blenis J (2004) ERK and p38 MAPK-activated protein kinases: a family of protein kinases with diverse biological functions. Microbiol Mol Biol Rev 68(2):320-344

49. Xie C, Kang J, Ferguson ME, Nagarajan S, Badger TM, Wu X (2011) Blueberries reduce pro-inflammatory cytokine TNF- $a$ and IL-6 production in mouse macrophages by inhibiting NF-KB activation and the MAPK pathway. Mol Nutr Food Res 55(10):1587-1591

50. Chu X, Ci X, He J, Wei M, Yang X, Cao Q, Li H, Guan S, Deng Y, Pang D (2011) A novel anti-inflammatory role for ginkgolide $B$ in asthma via inhibition of the ERK/MAPK signaling pathway. Molecules 16(9):7634-7648

51. Cheng YW, Chang CY, Lin KL, Hu CM, Lin CH, Kang JJ (2008) Shikonin derivatives inhibited LPS-induced NOS in RAW 264.7 cells via downregulation of MAPK/NF-KB signaling. J Ethnopharmacol 120(2):264-271

52. Marrero MB, Venema VJ, He H, Caldwell RB, Venema RC (1998) Inhibition by the JAK/STAT pathway of IFNY-and LPS-stimulated nitric oxide synthase induction in vascular smooth muscle cells. Biochem Biophys Res Commun 252(2):508-512

53. Morris R, Kershaw NJ, Babon JJ (2018) The molecular details of cytokine signaling via the JAK/STAT pathway. Protein Sci 27(12):1984-2009

\section{Publisher's Note}

Springer Nature remains neutral with regard to jurisdictional claims in published maps and institutional affiliations.

\section{Submit your manuscript to a SpringerOpen ${ }^{\circ}$ journal and benefit from:}

- Convenient online submission

- Rigorous peer review

- Open access: articles freely available online

- High visibility within the field

Retaining the copyright to your article

Submit your next manuscript at springeropen.com 